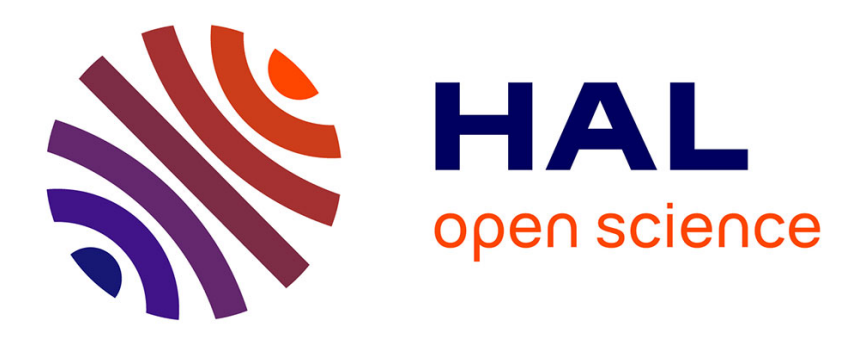

\title{
Formules analytiques pour le calcul du profil d'ions implantés
}

\author{
J.P. Eymery, A. Fnidiki
}

\section{To cite this version:}

J.P. Eymery, A. Fnidiki. Formules analytiques pour le calcul du profil d'ions implantés. Revue de Physique Appliquée, 1988, 23 (5), pp.925-932. 10.1051/rphysap:01988002305092500 . jpa-00245894

\section{HAL Id: jpa-00245894 https://hal.science/jpa-00245894}

Submitted on 1 Jan 1988

HAL is a multi-disciplinary open access archive for the deposit and dissemination of scientific research documents, whether they are published or not. The documents may come from teaching and research institutions in France or abroad, or from public or private research centers.
L'archive ouverte pluridisciplinaire HAL, est destinée au dépôt et à la diffusion de documents scientifiques de niveau recherche, publiés ou non, émanant des établissements d'enseignement et de recherche français ou étrangers, des laboratoires publics ou privés. 
Classification

Physics Abstracts

$02.60-61.70 \mathrm{~T}-61.80 \mathrm{~J}$

\title{
Formules analytiques pour le calcul du profil d'ions implantés
}

\author{
J. P. Eymery et A. Fnidiki
}

Laboratoire de Métallurgie Physique, U.A. 131 du C.N.R.S., 40 avenue du Recteur Pineau, 86022 Poitiers, France

(Reçu le 4 décembre 1987, révisé le 28 janvier 1988, accepté le 29 janvier 1988)

\begin{abstract}
Résumé. - Les deux premiers moments $m_{1}$ et $m_{2}$ (ou $\sigma$ ) décrivant le profil d'ions implantés dans un matériau cible à basse énergie peuvent maintenant être estimés à partir de formules analytiques simples. Dans cet article, nous proposons pour $m_{1}$ une expression qui fait seulement intervenir la fonction exponentielle intégrale et qui est valable pour deux potentiels interatomiques différents. En ce qui concerne le calcul de $\sigma$, nous donnons deux possibilités d'inégale difficulté ; la première est une expression dans laquelle $m_{2}$ s'exprime sous la forme d'un trinôme du second degré en $m_{1}$; la deuxième, plus simple, exploite la proportionnalité qui existe entre $m_{1}$ et $\sigma$.
\end{abstract}

\begin{abstract}
The first two moments $m_{1}$ and $m_{2}$ (or $\sigma$ ) describing the profile of ions implanted into a target material at low energies can now be estimated with the help of simple analytical formulae. In this paper, we propose for $m_{1}$ an expression which only uses the exponential integral function; moreover it appears to be valid for two different interatomic potentials. Concerning the estimation of $\sigma$, we suggest two possibilities of unequal difficulty ; first one is an expression in which $m_{2}$ appears as a quadratic trinomial ; the second one, which is more simple, uses the proportionality existing between $m_{1}$ and $\sigma$.
\end{abstract}

\section{Introduction.}

L'étude du profil des ions implantés dans un matériau cible est un sujet d'intérêt croissant essentiellement pour deux raisons; la première est que l'implantation ionique a reçu récemment de nombreuses applications et demeure encore en voie d'expansion ; la seconde est qu'il existe maintenant des techniques très précises pour déterminer les profils expérimentaux, citons par exemple le SIMS - secondary ion mass spectroscopy -, ce qui permet d'effectuer des comparaisons avec les profils théoriques. La majorité des approches pour le calcul des moments de la distribution de dopants est basée soit sur la résolution de l'équation de transport de Boltzmann [1], soit sur des simulations de type Monte-Carlo où les trajectoires des ions injectés dans la cible sont calculées à l'aide de la théorie des collisions binaires [2]. La principale difficulté, pour l'utilisation pratique de ces méthodes, réside dans la nécessité d'élaborer des algorithmes de calculs compliqués qui ont en fait conduit souvent à des tables numériques de moments, par exemple celles publiées par Littmark et Ziegler [3]. Une première tentative de simplification des calculs de profils a été effectuée par Biersack [4] avec l'algorithme PRAL (projected range algorithm); ceux-ci pouvaient alors être réalisés avec une calculatrice programmable.

Des recherches ont été également entreprises pour obtenir, avec le minimum de calculs et par suite le maximum de rapidité, les moments de la distribution et en particulier les deux premiers. Dans ce domaine, les formules analytiques conduisant à une application numérique réalisable avec une simple calculatrice de poche offrent de bonnes solutions. Le présent article traite de ce sujet et les résultats présentés ici font suite à ceux publiés récemment dans une lettre [5]. Ils s'inscrivent également au côté de ceux des références [4, 6-7]. Nous proposons des formules simples pour le calcul du premier moment $m_{1}$ et deux possibilités d'inégale difficulté pour le calcul du second moment $m_{2}$ et de l'écart-type $\sigma$. Comme dans l'article [5], elles sont établies en tenant seulement compte du ralentissement nucléaire, ce qui correspond à un domaine d'énergie réduite $\varepsilon_{0}$ tel que : $0,01 \leqslant \varepsilon_{0} \leqslant 0,2$. Avant de les aborder, nous rappellerons les notations employées ainsi que les résultats de [5] qui serviront de point de départ. 


\section{Notations et rappels.}

Les notations et relations employées sont les suivantes :

$E=$ énergie de l'ion, valeur initiale $E_{0}$; homologues en coordonnées réduites : $\varepsilon$ et $\varepsilon_{0}$;

$M_{1}, M_{2}=$ masses atomiques, respectivement de l'ion et de la cible ; $u=M_{2} / M_{1}$;

$Z_{1}, Z_{2}=$ numéros atomiques, respectivement de l'ion et de la cible ;

$e=$ charge de l'électron ;

$a_{0}=$ rayon de Bohr $\left(5,3 \times 10^{-9} \mathrm{~cm}\right)$;

$V=$ volume atomique $;=1 / V=$ densité atomique de la cible; expression du ralentissement nucléaire : $S_{\mathrm{n}}(E)=D S_{\mathrm{n}}^{\prime}(\varepsilon)$ avec :

$$
\begin{aligned}
D & =\left(4 \pi a Z_{1} Z_{2} e^{2} M_{1} N\right) /\left(M_{1}+M_{2}\right) \\
a & =0,8853 a_{0} /\left(Z_{1}^{2 / 3}+Z_{2}^{2 / 3}\right)^{1 / 2} ;
\end{aligned}
$$

relation entre $E$ et $\varepsilon: \quad \varepsilon=P E$ avec $P=a M_{2} /\left(Z_{1} Z_{2} e^{2}\left(M_{1}+M_{2}\right)\right)$. Dans l'article [5], nous avons utilisé une expression du ralentissement nucléaire de Wilson et al. [8] :

$$
S_{\mathrm{n}}^{\prime}(\varepsilon)=A \ln B \varepsilon /\left(B \varepsilon-(B \varepsilon)^{-c}\right)
$$

où $A, B$ et $C$ sont des constantes caractéristiques du potentiel interatomique (Molière, Bohr, ThomasFermi...) pour établir la relation suivante qui donne simplement le moment d'ordre 1 :

$$
m_{1}=Q /\left(A B\left(B \varepsilon_{0}\right)^{u / 2}\right)\left[E_{1}\left(T_{1}\right)-E_{1}\left(T_{2}\right)\right]
$$

où l'on a posé :

$$
\begin{aligned}
& Q=\left(M_{1}+M_{2}\right)^{2} / 4 \pi a^{2} M_{1} M_{2} N ; \\
& T_{1}=-(u / 2-C+1) \ln B \varepsilon_{0} ; \\
& T_{2}=-(u / 2+2) \ln B \varepsilon_{0} ;
\end{aligned}
$$

la fonction $E_{1}(x)$ est définie par :

$$
E_{1}(x)=\int_{x}^{+\infty} \exp (-t) / t \cdot \mathrm{d} t
$$

Nous avons également montré l'intérêt de cette formule en utilisant le potentiel de Molière [9] où les constantes $A, B$ et $C$ valent respectivement : 0,691 , 3,60 et 0,713 .

\section{Calcul du moment d'ordre 1 : utilisation de la} relation (2).

3.1 UTILISATION D'APPROXIMATIONS POUR LA FONCTION $E_{1}(x)$. - Pour éviter de consulter des tables pour déterminer $E_{1}\left(T_{1}\right)$ et $E_{1}\left(T_{2}\right)$, nous préconisons l'emploi des développements suivants. Dans le cas où $0<x \leq 1$, on peut se servir de l'approximation polynomiale [10] :

$$
\begin{array}{rl}
E_{1}(x)+\ln (x)=a_{0}+a_{1} & x+a_{2} x^{2}+a_{3} x^{3}+ \\
& +a_{4} x^{4}+a_{5} x^{5}+\eta(x)
\end{array}
$$

avec :

$$
\begin{aligned}
& a_{0}=-0,57721, a_{1}=-0,99999, a_{2}=-0,24991 \\
& a_{3}=0,05519, a_{4}=-0,00976, a_{5}=0,00107
\end{aligned}
$$

et : $|\eta(x)|<2 \times 10^{-7}$.

De même, dans le cas où $1 \leq x<+\infty$, il sera intéressant d'utiliser l'approximation rationnelle [11] :

$$
x \exp (x) E_{1}(x)=\frac{x^{2}+a_{1} x+a_{2}}{x^{2}+b_{1} x+b_{2}}+\eta(x)
$$

avec :

$$
\begin{aligned}
& a_{1}=2,334733, a_{2}=0,250621, b_{1}=3,330657, \\
& b_{2}=1,681534 \text { et }|\eta(x)|<5 \times 10^{-5} .
\end{aligned}
$$

On notera que pour des calculs approchés, on peut arrêter le polynôme de la relation (3) au terme d'ordre 3. Au contraire, si l'on désire des résultats plus précis, on peut non seulement l'utiliser intégralement, mais encore remplacer l'expression (4) par l'approximation :

$$
\begin{aligned}
& x \exp (x) E_{1}(x)= \\
& =\frac{x^{4}+a_{1} x^{3}+a_{2} x^{2}+a_{3} x+a_{4}}{x^{4}+b_{1} x^{3}+b_{2} x^{2}+b_{3} x+b_{4}}+\eta(x)
\end{aligned}
$$

et : $|\eta(x)|<2 \times 10^{-8}$.

Nous avons testé la formule (2) en utilisant le potentiel de Molière et, pour le calcul de $E_{1}(x)$, les expressions (3) ou (4) suivant la position de $x$ par rapport à 1 . Les résultats ont été tout d'abord comparés avec ceux obtenus par le programme TRIM (transport of ions in matter) de Biersack [12] ou plus exactement la nouvelle version de celui-ci, TRIM 86, adaptée pour les microordinateurs. Rappelons que les programmes TRIM sont basés sur une méthode de Monte-Carlo et qu'ils demandent un temps de calcul relativement long, par exemple de l'ordre d'une demi-heure pour 500 ions incidents. Les couples ions-cibles choisis pour effectuer la comparaison sont $\mathrm{Bi} \rightarrow \mathrm{Be}$ et $\mathrm{Te} \rightarrow \mathrm{LiF}$ et les résultats sont reportés sur les figures $1 \mathrm{a}$ et $1 \mathrm{~b}$ respectivement pour la gamme d'énergie réduite retenue, soit : $0,01 \leq \varepsilon_{0} \leq 0,2$. Sur la première figure (cas de $\mathrm{Bi} \rightarrow \mathrm{Be}$ ), on constate un très bon accord entre la formule (2) et le programme TRIM ; l'écart, qui est pratiquement nul au début de la gamme d'énergie, n'excède pas $5 \%$ en fin de gamme. Sur la seconde figure (cas de $\mathrm{Te} \rightarrow \mathrm{LiF}$ ), l'accord est encore bon entre les deux modes de calculs ; toutefois, TRIM donne des résultats légèrement supérieurs à ceux de 

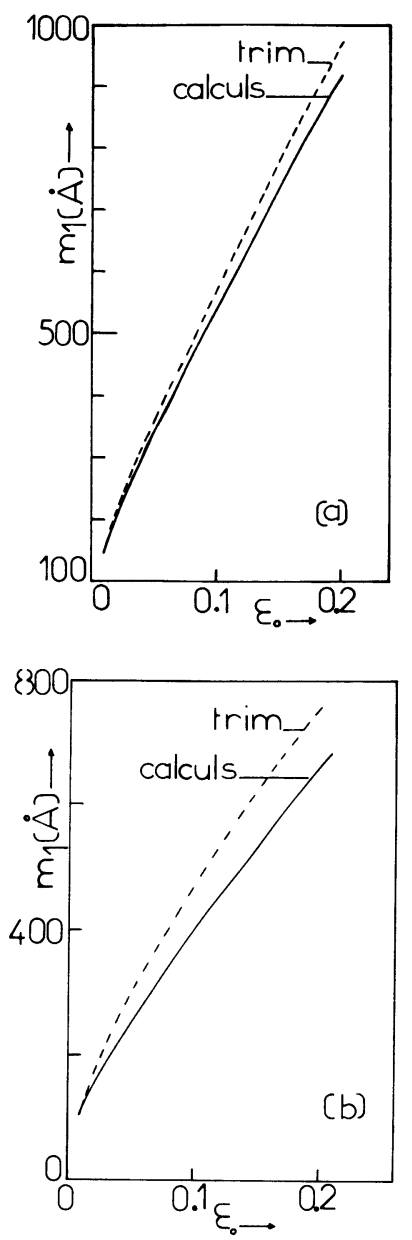

Fig. 1. - Variations de $m_{1}$ en fonction de l'énergie réduite $\varepsilon_{0}$ et comparaison entre les calculs et le programme TRIM 86: (a) implantation $\mathrm{Bi} \rightarrow \mathrm{Be}$; (b) implantation $\mathrm{Te} \rightarrow \mathrm{LiF}$.

[Variations in $m_{1}$ as a function of reduced energy $\varepsilon_{0}$ and comparison between present calculations and TRIM 86 computer code : (a) $\mathrm{Bi} \rightarrow$ Be implantation ; (b) $\mathrm{Te} \rightarrow \mathrm{LiF}$ implantation.]

la formule (2) et la différence atteint $13 \%$ pour $\varepsilon_{0}=0,2$. Ceci se produit bien que TRIM tienne compte du ralentissement électronique; dans la mesure où les calculs de cet article ne le prennent pas en compte, on pourrait s'attendre à ce que les parcours donnés par la relation (2) soient supérieurs à ceux donnés par TRIM ; or, il n'en est rien, sans doute parce que TRIM contient lui aussi des approximations et que le choix du potentiel interatomique conditionne de façon importante la valeur de $m_{1}$ déduite de la formule (2) (cf. paragraphe 3.2). Précisons également que pour le composé $\mathrm{LiF}$, les seules approximations employées ont consisté à prendre pour masse et numéro atomiques la moyenne arithmétique de ceux des constituants.

Après avoir comparé les résultats donnés par la formule (2) avec ceux d'un programme théorique, il est intéressant de les comparer également à ceux déduits de l'expérience. C'est précisément l'objet du tableau I qui a été établi dans le cas d'une cible de silicium. On peut y constater que les parcours déduits de la relation (2) soutiennent une bonne comparaison avec ceux qui ont été déterminés expérimentalement dans les références [13-15].

Tableau I. - Comparaison entre les parcours $\left(m_{1}\right)$ expérimentaux et théoriques dans le cas du silicium implanté à basse énergie.

[Comparison between theoretical and experimental ranges $\left(m_{1}\right)$ in the case of silicon implanted at low energy.]

\begin{tabular}{|c|c|c|c|c|}
\hline $\begin{array}{c}\text { ion } \\
\text { incident }\end{array}$ & $\begin{array}{c}\text { énergie } \\
(\mathrm{keV})\end{array}$ & $\begin{array}{l}m_{1}(\AA) \\
\text { expérimental }\end{array}$ & références & $\begin{array}{l}m_{1}(\AA): \\
\text { formule } \\
(2)\end{array}$ \\
\hline $\mathrm{Sb}$ & 45 & 250 & {$[13]$} & 245 \\
$\mathrm{As}$ & 15 & $120 \pm 8$ & {$[14]$} & 123 \\
$\overline{\mathrm{Xe}}$ & 10 & $106 \pm 5$ & - & 94 \\
$\overline{\mathrm{Kr}}$ & 40 & $\sim 200$ & {$[15]$} & 226 \\
& 8 & $\sim 115$ & - & 100 \\
& $\sim 100$ & - & 83 \\
\hline
\end{tabular}

3.2 RÓle du POTENTIEL INTERATOMIQUe. - On se propose ici de tester la formule (2) dans laquelle on emploie les approximations (3) et (4) vis-à-vis de tous les potentiels interatomiques cités dans la référence [8], soit respectivement : Thomas-Fermi, Molière, Bohr, $\mathrm{Kr}-\mathrm{C}$ et average. Les calculs de la référence [5] et ceux du paragraphe précédent ont déjà mis en relief l'intérêt du potentiel de Molière [9]. En fait, il s'avère que le potentiel de Thomas-Fermi (constantes $A=0,494, B=1,357$, $C=0,504$ ) donne également de bons résultats. Pour s'en persuader, on peut se reporter à la figure 2 qui

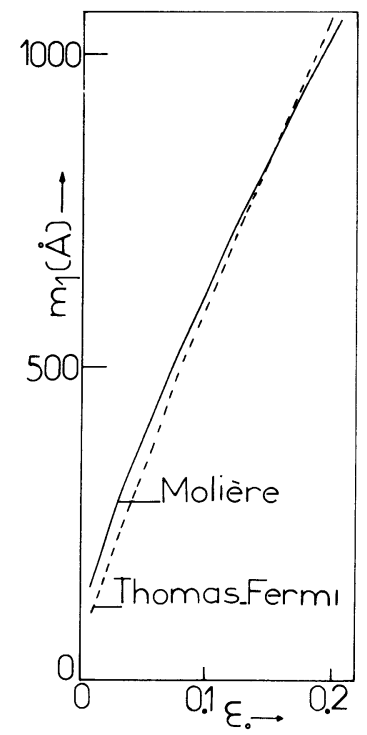

Fig. 2. - Variations de $m_{1}$ en fonction de l'énergie réduite $\varepsilon_{0}$ : comparaison entre deux potentiels interatomiques dans le cas de $\mathrm{Ag}$ implanté dans $\alpha-\mathrm{Al}_{2} \mathrm{O}_{3}$.

[Variations in $m_{1}$ as a function of reduced energy $\varepsilon_{0}$ : comparison between two interatomic potentials in the case of $\mathrm{Ag}$ implanted into $\alpha-\mathrm{Al}_{2} \mathrm{O}_{3}$.] 
compare le potentiel de Molière à celui de ThomasFermi dans le cas de $\mathrm{Ag}$ implanté dans $\alpha-\mathrm{Al}_{2} \mathrm{O}_{3}$. Pour $\varepsilon_{0}<0,1$, on observe un écart qui n'excède pas $13 \%$ et, pour $\varepsilon_{0}>0,1$, il n'existe pratiquement pas de différence. Les trois autres potentiels surestiment $m_{1}$ si bien qu'il n'y a pas lieu de les retenir; à titre d'exemple, pour $\varepsilon_{0}=0,01$, le potentiel $\mathrm{Kr}-\mathrm{C}$ conduit à une valeur de $m_{1}$ double de celle calculée avec le potentiel de Thomas-Fermi. Les résultats décrits ici se sont avérés aussi valables pour tous les autres couples ions-cibles étudiés. Précisons enfin que les approximations employées pour le calcul de $M_{2}$ et $Z_{2}$ dans le cas du composé $\alpha-\mathrm{Al}_{2} \mathrm{O}_{3}$ sont analogues à celles employées pour $\mathrm{LiF}$.

3.3 SimplifiCATION DE LA FORMULE (2). - L'utilisation de la formule (2) montre que souvent le terme $E_{1}\left(T_{2}\right)$ est petit devant $E_{1}\left(T_{1}\right)$ et par suite, peut être négligé dans certains cas que nous allons préciser. D'une façon qualitative, on peut expliquer ceci de la manière suivante; on vérifie aisément que l'on a toujours $T_{2}>3 T_{1}$ avec le potentiel de Molière et la conditions $u<1$; d'autre part, la fonction $E_{1}(x)$ est fortement décroissante pour $0<x \leq 3$ (voir son graphe dans la Réf. [5]); il en résulte donc des circonstances où $E_{1}\left(T_{2}\right) \ll E_{1}\left(T_{1}\right)$ et par suite la formule (2) peut se réduire à :

$$
m_{1}=Q /\left(A B\left(B \varepsilon_{0}\right)^{u / 2}\right) . E_{1}\left(T_{1}\right) .
$$

La figure 3 permet de préciser le domaine de validité de la formule (6). Elle correspond à une implantation de Xe dans $\mathrm{Ge}$ et a été tracée en utilisant le potentiel de Molière. On peut constater que la

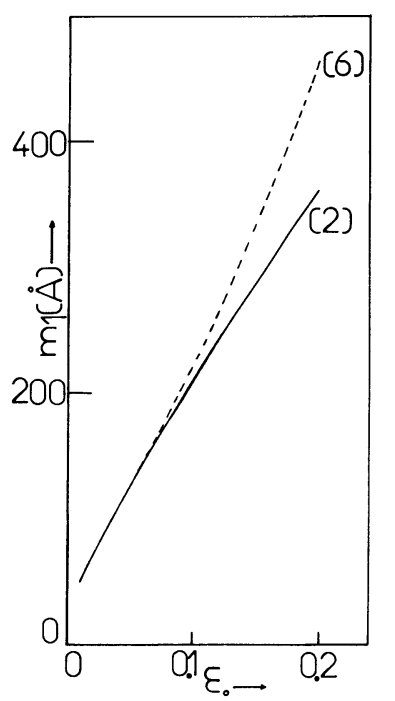

Fig. 3. - Variations de $m_{1}$ en fonction de l'énergie réduite $\varepsilon_{0}$ : comparaison entre la formule complète (2) et la formule simplifiée (6) (implantation : $\mathrm{Xe} \rightarrow \mathrm{Ge}$ ).

[Variations in $m_{1}$ as a function of reduced energy $\varepsilon_{0}$ : comparison between complete formula (2) and simplified formula (6) (Xe $\rightarrow \mathrm{Ge}$ implantation).] formule (2) et la formule (6) diffèrent très peu l'une de l'autre pour $\varepsilon_{0}<0,1$; au contraire, l'écart s'accroît nettement dans le domaine $\varepsilon_{0}>0,1$, et la formule (6) donne toujours un résultat supérieur à celui de la formule (2). Toutefois, comme le programme TRIM conduit également à une valeur de $m_{1}$ légèrement plus forte que celle calculée par la formule (2), il sera possible d'obtenir des résultats raisonnables avec la relation (6) jusqu'à $\varepsilon_{0}=0,15$. $\mathrm{Au}$ vu de ce qui précède et des calculs effectués sur d'autres couples ions-cibles, nous préconisons donc l'emploi de la formule simplifiée (6) dans le domaine $0,01 \leq \varepsilon_{0} \leq 0,15$.

\section{Calcul du moment d'ordre 2 : formule analytique.}

Dans notre précédent article [5], nous avions déjà proposé une première formule analytique pour le calcul de $m_{2}$. En partant de la même expression, nous en proposons ici une autre; sa formulation et sa démonstration sont plus élégantes puisque nous montrons que $m_{2}$ peut s'exprimer sous la forme d'un trinôme du second degré en $m_{1}$; de plus, son emploi pour les calculs numériques sera plus rapide.

La relation de départ est comme dans la référence [5] :

$$
\begin{aligned}
m_{2}=2(Q / A B)^{2} \int_{0}^{x_{0}} x_{2}^{u / 2}\left(x_{2}-x_{2}^{-c}\right) / \ln x_{2} \times \\
\times\left[\int_{x_{2}}^{x_{0}} x_{1}^{-u / 2}\left(x_{1}-x_{1}^{-c}\right) / \ln x_{1} \cdot \mathrm{d} x_{1}\right] \mathrm{d} x_{2}
\end{aligned}
$$

avec : $x=B \varepsilon$.

Tout d'abord, nous posons :

$$
x_{1}^{-u / 2}=x_{1}^{u / 2} \cdot x_{1}^{-u}
$$

et travaillons avec l'hypothèse où $u \cdot \ln x$ est petit, ce qui permet d'écrire : $x_{1}^{-u} \sim 1-u \cdot \ln x_{1}$ : de plus, on note :

$$
G(x)=x^{u / 2}\left(x-x^{-c}\right) / \ln x .
$$

L'expression (7) devient alors :

$$
\begin{aligned}
& m_{2}=2(Q / A B)^{2}\left[\int_{0}^{x_{0}} G\left(x_{2}\right)\left(\int_{x_{2}}^{x_{0}} G\left(x_{1}\right) \mathrm{d} x_{1}\right) \times\right. \\
& \left.\quad \times \mathrm{d} x_{2}-u \int_{0}^{x_{0}} G\left(x_{2}\right)\left(\int_{x_{2}}^{x_{0}} \ln x_{1} G\left(x_{1}\right) \mathrm{d} x_{1}\right) \mathrm{d} x_{2}\right] .
\end{aligned}
$$

L'équation (8) peut alors s'écrire à l'aide de deux intégrales $I_{1}$ et $I_{2}$ :

$$
m_{2}=2(Q / A B)^{2}\left(I_{1}-u I_{2}\right)
$$

qu'il est nécessaire de calculer successivement. 
Calcul de $I_{1}=\int_{0}^{x_{0}} G\left(x_{2}\right)\left(\int_{x_{2}}^{x_{0}} G\left(x_{1}\right) \mathrm{d} x_{1}\right) \mathrm{d} x_{2}$ :

$$
\begin{aligned}
I_{1}=\int_{0}^{x_{0}} G\left(x_{2}\right)( & \left.\int_{0}^{x_{0}} G\left(x_{1}\right) \mathrm{d} x_{1}\right) \mathrm{d} x_{2}- \\
& -\int_{0}^{x_{0}} G\left(x_{2}\right)\left(\int_{0}^{x_{2}} G\left(x_{1}\right) \mathrm{d} x_{1}\right) \mathrm{d} x_{2}
\end{aligned}
$$$$
I_{1}=\left(\int_{0}^{x_{0}} G\left(x_{1}\right) \mathrm{d} x_{1}\right)^{2}-1 / 2\left(\int_{0}^{x_{0}} G\left(x_{1}\right) \mathrm{d} x_{1}\right)^{2}=
$$$$
=1 / 2\left(\int_{0}^{x_{0}} G\left(x_{1}\right) \mathrm{d} x_{1}\right)^{2} \text {. }
$$

Compte tenu de la relation: $\int_{0}^{x_{0}} x^{\alpha} / \ln x \cdot \mathrm{d} x=-$ $E_{1}\left(-(\alpha+1) \ln x_{0}\right)$, on trouve aisément :

$$
\begin{aligned}
\int_{0}^{x_{0}} G\left(x_{1}\right) \mathrm{d} x_{1} & =E_{1}\left(T_{1}\right)-E_{1}\left(T_{2}\right) \\
& =(A B / Q) x_{0}^{u / 2} m_{1},
\end{aligned}
$$

ce qui permet d'exprimer $I_{1}$ en fonction de $m_{1}$ de la façon suivante : $I_{1}=1 / 2(A B / Q)^{2} x_{0}^{u} m_{1}^{2}$.

On calcule maintenant :

$$
I_{2}=\int_{0}^{x_{0}} G\left(x_{2}\right)\left(\int_{x_{2}}^{x_{0}} \ln x_{1} G\left(x_{1}\right) \mathrm{d} x_{1}\right) \mathrm{d} x_{2} .
$$

L'expression entre parenthèses conduit facilement à :

$$
\begin{aligned}
\left(x_{0}^{u / 2+2} /(u / 2+2)-\right. & \left.x_{0}^{u / 2-c+1} /(u / 2-C+1)\right) \\
& -\left(x_{2}^{u / 2+2} /(u / 2+2)\right. \\
& \left.-x_{2}^{u / 2-c+1} /(u / 2-C+1)\right) .
\end{aligned}
$$

On constate que le premier terme (soit $K_{1}$ ) ne dépend pas de $x_{2}$; par contre, le second (soit $\left.K_{2}\left(x_{2}\right)\right)$ en dépend. L'intégrale $I_{2}$ s'écrit alors :

$$
I_{2}=K_{1} \int_{0}^{x_{0}} G\left(x_{2}\right) \mathrm{d} x_{2}-\int_{0}^{x_{2}} G\left(x_{2}\right) K_{2}\left(x_{2}\right) \mathrm{d} x_{2}
$$

que nous notons :

$$
I_{2}=K_{1} J_{1}-J_{2} .
$$

Il est donc nécessaire de calculer maintenant les intégrales $J_{1}$ et $J_{2}$.

L'obtention de $J_{1}$ est immédiate compte tenu des résultats précédents :

$$
J_{1}=E_{1}\left(T_{1}\right)-E_{1}\left(T_{2}\right)=(A B / Q) x_{0}^{u / 2} m_{1} .
$$

Le calcul de $J_{2}$ fait intervenir seulement la fonction $E_{1}(x)$. On trouve aisément :

$$
\begin{aligned}
J_{2}=\frac{E_{1}\left(-(u+4) \ln x_{0}\right)}{u / 2+}-\frac{E_{1}\left(-(u-2 C+2) \ln x_{0}\right)}{u / 2-C+1} \\
+\frac{u-C+3}{(u / 2+2)(u / 2-C+1)} \times \\
\times E_{1}\left(-(u-C+3) \ln x_{0}\right) . \quad(10)
\end{aligned}
$$

Le report dans la relation (9) conduit à :

$$
\begin{aligned}
m_{2}=2(Q / A B)^{2}[ & (A B / Q)^{2} x_{0}^{u} m_{1}^{2}-\times \\
& \times u\left\{\left(\frac{x_{0}^{u / 2+2}}{u / 2+2}-\frac{x_{0}^{u / 2-c+1}}{u / 2-C+1}\right)\right. \\
& \left.\left.\times(A B / Q) x_{0}^{u / 2} m_{1}-J_{2}\right\}\right] .
\end{aligned}
$$

Finalement $m_{2}$ s'écrit de la façon suivante :

$$
\begin{aligned}
& m_{2}=x_{0}^{u} m_{1}^{2}- 2(Q / A B) \times \\
& \times u\left(\frac{x_{0}^{u+2}}{u / 2+2}-\frac{x_{0}^{u-c+1}}{u / 2-C+1}\right) m_{1}+ \\
&+2(Q / A B)^{2} u J_{2}
\end{aligned}
$$

où $J_{2}$ est donné par la relation (10). Comme annoncé, $m_{2}$ se présente donc sous la forme d'un trinôme du second degré en $m_{1}$ et seul le calcul du terme constant nécessite l'emploi de la fonction $E_{1}(x)$; encore celle-ci n'est-elle nécessaire que trois fois. Dans la pratique, il est en fait plus intéressant de calculer $\sigma=\left(m_{2}-m_{1}^{2}\right)^{1 / 2}$.

Nous avons testé la formule (11) en utilisant comme précédemment le potentiel de Molière et pour le calcul de $E_{1}(x)$, les expressions (3) et (4). Les exemples choisis sont les mêmes qu'au paragraphe 3.1 , soit $\mathrm{Bi} \rightarrow \mathrm{Be}$ et $\mathrm{Te} \rightarrow \mathrm{LiF}$; les résultats sont également comparés à ceux déduits du programme TRIM. Les comparaisons sont reportées sur les figures $4 \mathrm{a}$ et $4 \mathrm{~b}$ respectivement pour la gamme d'énergie réduite : $0,01 \leqslant \varepsilon_{0} \leqslant 0,2$. Sur la figure $4 \mathrm{a}$ (cas de $\mathrm{Bi} \rightarrow \mathrm{Be}$ ), on constate un accord satisfaisant entre le calcul analytique de $\sigma$ et le programme TRIM, l'écart entre les deux atteignant $28 \%$ en fin de gamme. Il en est de même sur la figure $4 b$ (cas de $\mathrm{Te} \rightarrow \mathrm{LiF}$ ) où l'écart en fin de gamme n'est que de $18 \%$. D'une manière générale, il s'avère que la formule (11) donne des résultats légèrement supérieurs à ceux de TRIM. Comme au paragraphe 3.1, il est nécessaire maintenant d'effectuer une comparaison entre la formule (11) et l'expérience. La référence [16] qui concerne une implantation de $\mathrm{Kr}$ dans $\mathrm{Si}$ à $40 \mathrm{keV}\left(\varepsilon_{0}=0,16\right)$ en fournit la possibilité ; expérimentalement on peut déduire un écarttype de l'ordre de $140 \AA$ alors que la formule (11) conduit à $95 \AA$. Les écarts que l'on constate entre le programme TRIM, la formule (11) et l'expérience reflètent une certaine dispersion des valeurs de $\sigma$ qu'il est habituel de constater dans la littérature mais qui n'altèrent en rien la validité de la relation (11). Il reste cependant à préciser les restrictions apportées 

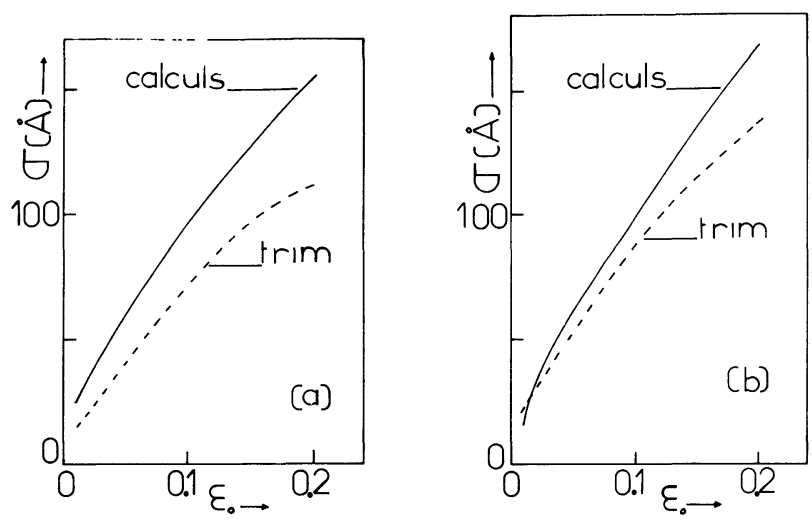

Fig. 4. - Variations de $\sigma$ en fonction de l'énergie réduite $\varepsilon_{0}$ et comparaison entre les calculs et le programme TRIM 86: (a) implantation $\mathrm{Bi} \rightarrow \mathrm{Be}$, (b) implantation $\mathrm{Te} \rightarrow \mathrm{LiF}$.

[Variations in $\sigma$ as a function of reduced energy $\varepsilon_{0}$ and comparison between present calculations and TRIM 86 computer code : (a) $\mathrm{Bi} \rightarrow \mathrm{Be}$ implantation, (b) $\mathrm{Te} \rightarrow \mathrm{LiF}$ implantation.]

par la condition $u . \ln x_{0}$ petit nécessaire pour l'intégration de la relation (7) dans le domaine: $0,01 \leqslant \varepsilon_{0} \leqslant 0,2$. Au voisinage de la borne inférieure $\varepsilon_{0}=0,01$, il est impératif que $u$ vérifie la condition : $u \ll 0,3$; en fait, l'expérience montre que $u$ est tolérable jusqu'à environ 0,15 . De même au voisinage de la borne supérieure $\varepsilon_{0}=0,2, u$ doit vérifier : $u \ll 3$ et en pratique, on peut tolérer des valeurs de $u$ allant jusqu'à 1 ou 1,5 .

\section{Calcul de l'écart type : proportionnalité entre $\boldsymbol{m}_{1}$ et $\boldsymbol{\sigma}$.}

Les calculs de moments d'ordre 2 peuvent tirer parti de la constatation suivante qu'il est bon de noter pour apporter des solutions extrêmement rapides : $m_{1}$ et $\sigma$ s'avèrent être proportionnels dans la gamme d'énergie réduite retenue. Ceci peut être à la fois démontré analytiquement et vérifié, par exemple à l'aide des programmes PRAL et TRIM.

De l'ancienne théorie L.S.S. [17], on peut déjà extraire :

$$
\sigma=(\gamma / 6)^{1 / 2} m_{1} \text { avec } \gamma=4 M_{1} M_{2} /\left(M_{1}+M_{2}\right)^{2},
$$

ce qui conduit à :

$$
\sigma=0,82 u^{1 / 2} /(1+u) \cdot m_{1} .
$$

De même, des formules analytiques de Biersack [4], on peut déduire :

$$
\sigma=\left(u / 2 B^{\prime}\right) /\left(1+u / B^{\prime}\right)^{1 / 2} \cdot m_{1}
$$

où la constante $B^{\prime}$ est comprise entre 0,4 et 0,6 . Pour la valeur intermédiaire de 0,5 , il vient :

$$
\sigma=u /(1+2 u)^{1 / 2} \cdot m_{1} \text {. }
$$

Ensuite des relations établies par Martan et Mulak [6], on peut tirer :

$$
\begin{aligned}
m_{2} & =m_{1}^{2}(1+0,6377 u) \\
\sigma & =0,8 u^{1 / 2} \cdot m_{1} .
\end{aligned}
$$

Enfin, le récent article de Fink et al. [7] conduit à :

$$
\sigma=0,6456 \exp \left(-0,0564 \ln ^{2}(10 u)\right) \cdot m_{1} .
$$

Les formules (12-15) montrent donc que $\sigma$ peut se mettre sous la forme : $\sigma=f(u) \cdot m_{1}$ où $f(u)$ est une fonction simple de $u$. La proportionnalité entre $m_{1}$ et $\sigma$ peut également être vérifiée à l'aide du programme TRIM 86 ; la figure 5 le montre pour l'implantation $\mathrm{Kr} \rightarrow \mathrm{Ag}$ d'une part et $\mathrm{Cu} \rightarrow \mathrm{Ti}$ d'autre part, dans le domaine d'énergie $0,01 \leq \varepsilon_{0} \leq 0,2$. Des constatations analogues ont été effectuées avec le progamme PRAL sur les couples ions-cibles suivants $: \mathrm{Au} \rightarrow \mathrm{Si}, \mathrm{Te} \rightarrow \mathrm{Al}, \mathrm{Au} \rightarrow \mathrm{Cu}$ et $\mathrm{Xe} \rightarrow \mathrm{Ge}$.

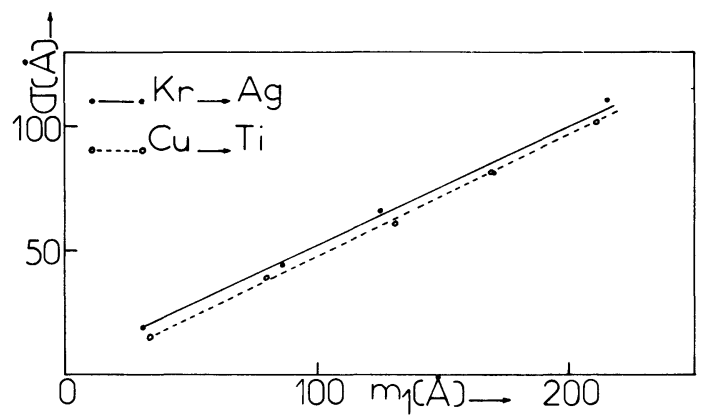

Fig. 5. - Proportionnalité entre $m_{1}$ et $\sigma$ pour $0,01 \leq \varepsilon_{0} \leq 0,2$ (implantations $\mathrm{Kr} \rightarrow \mathrm{Ag}$ et $\mathrm{Cu} \rightarrow \mathrm{Ti}$ ). [Proportionality between $m_{1}$ and $\sigma$ in the $0.01 \leq \varepsilon_{0} \leq 0.02$ range $(\mathrm{Kr} \rightarrow \mathrm{Ag}$ and $\mathrm{Cu} \rightarrow \mathrm{Ti}$ implantations).]

En réalité, la fonction $f$ devrait dépendre non seulement de $M_{1}$ et $M_{2}$, mais aussi de $Z_{1}$ et $Z_{2}$. Sur une série d'exemples concrets, nous avons vérifié qu'en fait la dépendance vis-à-vis de $Z_{1}$ et $Z_{2}$ était faible, aussi l'écriture de $\sigma$ sous la forme : $\sigma=f(u) \cdot m_{1}$ est une bonne approximation. Pour notre part, nous avons également cherché à déterminer des fonctions $f(u)$ sous la forme de lois puissance du type : $f(u)=\alpha u^{\beta}$ où $\alpha$ et $\beta$ sont des constantes. Pour cela, nous avons sélectionné 8 valeurs de $u$ comprises entre 0 et 1,3 puis le tracé de $\ln \left(\mathrm{d} \sigma / \mathrm{d} m_{1}\right)$ en fonction de $\ln (u)$ a permis de déduire :

i) $\alpha=0,5$ et $\beta=0,42$ avec le programme TRIM,

ii) $\alpha=0,45$ et $\beta=0,5$ avec le programme PRAL.

Nous proposons donc les relations :

$$
\text { et } \quad \begin{array}{ll}
\sigma & =0,5 u^{0,42} \cdot m_{1} \\
\text { en } & =0,45 u^{1 / 2} \cdot m_{1} .
\end{array}
$$


Présentant un formalisme voisin de la relation (14), elles s'en démarquent cependant par la valeur du coefficient $\alpha$ qui est ici plus faible.

Sur l'ensemble des formules (12-17), il reste à effectuer des comparaisons et éventuellement des choix. Pour cela, nous avons tracé, sur la figure 6, les fonctions $f(u)$ de ces formules dans le domaine : $0 \leq u \leq 1,5$. On y constate que les fonctions issues des relations (12-13) et (15-17) forment un faisceau de courbes relativement cohérent pour $0,5 \leq u \leq 1,5$; par contre, celles des relations (14) et (15) s'écartent ássez notablement des autres pour $u>0,5$ et $\mathrm{u}<0,5$ respectivement. Pour ces raisons, nous préconiserons surtout l'emploi des relations (12-13) et (16-17) dans le domaine de valeurs de $u$ et $\varepsilon_{0}$ retenu.

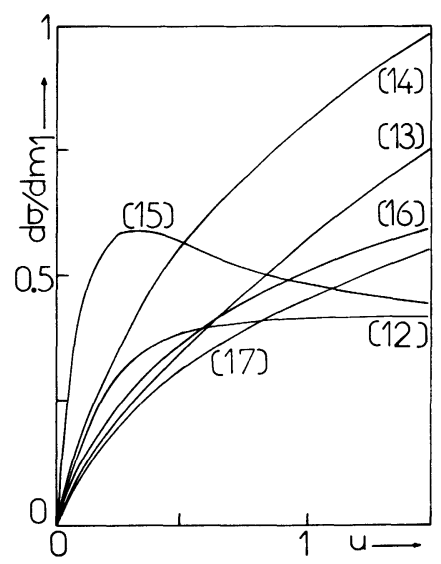

Fig. 6. - Graphes des fonctions $\mathrm{d} \sigma / \mathrm{d} m_{1}=f(u)$ suivant les relations (12-17).

[Plot of the $\mathrm{d} \sigma / \mathrm{d} m_{1}=f(u)$ functions according to relations (12-17).]

On retiendra essentiellement de ce paragraphe que, connaissant $m_{1}$, on peut très rapidement calculer une valeur approchée, mais cependant satisfai- sante, de $\sigma$ grâce à la linéarité entre $m_{1}$ et $\sigma$ et en utilisant de préférence les formules $(12,13)$ et $(16$, 17).

\section{Conclusion.}

Cet article a été rédigé avec le souci majeur de simplifier le calcul des deux premiers moments de la distribution des ions implantés dans une cible à faible énergie $\left(0,01 \leqslant \varepsilon_{0} \leqslant 0,2\right)$; les formules proposées conduisent à des applications numériques immédiates réalisables avec des calculatrices électroniques de poche. Pour le calcul du moment d'ordre 1 , nous avons travaillé à partir de la formule (2) démontrée antérieurement [5]. Il s'est avéré que des approximations polynomiales ou rationnelles pour la fonction exponentielle intégrale étaient satisfaisantes; de plus, la formule (2) peut être utilisée soit avec le potentiel de Molière, soit avec celui de ThomasFermi, ce qui en consolide son intérêt ; enfin, pour $0,1 \leqslant \varepsilon_{0} \leqslant 0,15$, on peut omettre un terme, ce qui conduit à la formule simplifiée (6). Pour le calcul de $m_{2}$ et de l'écart-type $\sigma$, nous proposons tout d'abord une formule élégante (11) dans laquelle $m_{2}$ apparaît comme un trinôme du second degré en $m_{1}$; dans ce formalisme, seul le terme constant dépend de la fonction exponentielle intégrale et les résultats obtenus soutiennent une bonne comparaison avec ceux de TRIM 86 qui est un programme très élaboré. Compte tenu des restrictions qui apparaissent alors sur les valeurs de $u$, nous avons également proposé une autre méthode qui exploite la proportionnalité existant entre $m_{1}$ et $\sigma$; il en est alors issu les relations (16) et (17) dont nous suggérons l'emploi au côté des relations (12) et (13).

\section{Remerciements.}

Les auteurs remercient l'équipe de recherche de J. P. Biersack (Berlin) qui a fourni au laboratoire les programmes de calcul utilisés.

\section{Bibliographie}

[1] Littmark, U. et Ziegler, J. F., Phys. Rev. A 23 (1981) 64 et références incluses.

[2] Robinson, J. E., KwoK, K. K. et Thompson, D. A., Nucl. Instrum. Methods 132 (1976) 667.

[3] Littmark, U. et Ziegler, J. F., Handbook of Range Distribution for Energetic Ions in All Elements, Vol. 6 of The Stopping and Ranges of Ions in Matter, Ed. J. F. Ziegler (Pergamon Press, New York) 1980.

[4] Biersack, J. P., Nucl. Instrum. Methods 182/183 (1981) 199.

[5] EYMERY, J. P. et FNIDIKI, A., Europhys. Lett. 4, 2 (1987) 223.
[6] Martan, J. et MulaK, A., Mat. Sci. Eng. 69 (1985) 21.

[7] Fink, D., Biersack, J. P., SANChEZ, P. F. et FICHTNER, P. F., Radiat. Eff. 103 (1987) 89.

[8] Wilson, W. D., Haggmark, L. G. et Biersack, J. P., Phys. Rev. B 15 (1977) 2458.

[9] Molière, G., Z. Naturforsch. Teil A 2 (1947) 133.

[10] Allen, E. E., Note 169, MTAC 8 (1954) 240.

[11] Hastings, C., Approximations for digital computers (Princeton Univ. Press, Princeton, N.J.) 1955.

[12] Biersack, J. P. et Haggmark, L. G., Nucl. Instrum. Methods 174 (1980) 257. 
[13] Ryssel, H., Ion Implantation Techniques, Eds. Ryssel and Glawischnig (Springer Verlag, Berlin) 1982, p. 117.

[14] Scanlon, P. J., Barfoot, K. M., Skensved, P. et WhitTon, J. L., Nucl. Instrum. Methods in P.R. B 7/8 (1985) 342 .
[15] Menzel, N. et WittmaAcK, K. Nucl. Instrum. Methods in P.R. B 7/8 (1985) 366.

[16] Paine, B. M. et Nicolet, M. A., Nucl. Instrum. Methods 209/210 (1983) 173.

[17] Lindhard, J., Scharf, M. et SchiøtT, H. E., Mat. Fys. Medd. Dan. Vid. Selsk 33 (1963) 14. 